\title{
A Note on Non-Degenerate Integer Programs with Small Sub-Determinants
}

\author{
S. Artmann ${ }^{1}$, F. Eisenbrand ${ }^{2}$, C. Glanzer ${ }^{1}$, T. Oertel $^{3}$, \\ S. Vempala ${ }^{4}$, and R. Weismantel ${ }^{1}$ \\ ${ }^{1}$ Swiss Federal Institute of Technology, Zürich (ETH Zürich) \\ ${ }^{2}$ École Polytechnique Fédérale de Lausanne (EPFL) \\ ${ }^{3}$ Cardiff University \\ ${ }^{4}$ Georgia Institute of Technology
}

October 1, 2018

\begin{abstract}
The intention of this note is two-fold. First, we study integer optimization problems in standard form defined by $A \in \mathrm{z}^{m \times n}$ and present an algorithm to solve such problems in polynomial-time provided that both the largest absolute value of an entry in $A$ and $m$ are constant. Then, this is applied to solve integer programs in inequality form in polynomial-time, where the absolute values of all maximal sub-determinants of $A$ lie between 1 and a constant.
\end{abstract}

\section{Introduction}

Let $A \in \mathbb{Z}^{m \times n}$ be a matrix such that all of its entries are bounded in absolute value by an integer $\Delta$. Assume that for each row index $i, \operatorname{gcd}\left(A_{i, \cdot}\right)=1$. We call the determinant of an $(n \times n)$-submatrix of $A$ an $(n \times n)$-sub-determinant of $A$. Let

$$
\delta_{\max }(A):=\max \{|d|: d \text { is an }(n \times n) \text {-sub-determinant of } A\} .
$$

We study the complexity of an integer programming problem in terms of the parameter $\Delta$ when presented in standard form (11). Moreover, we study integer programming problems in inequality form (2) that are associated with the matrix $A$ whose 'complexity' is measured by the parameter $\delta_{\max }(A)$.

$$
\begin{aligned}
& \max \left\{c^{T} x: A x=b, x \geqslant 0, x \in \mathbb{Z}^{n}\right\}, \\
& \max \left\{c^{T} x: A x \leq b, x \in \mathbb{Z}^{n}\right\} .
\end{aligned}
$$


It is known that when the absolute value of all sub-determinants of $A$ is bounded by one, $A$ is totally unimodular and the integer programs (11) and (2) are polynomially solvable. This concept of total unimodularity was pioneered by the works of Hoffman, Kruskal, Veinott, Dantzig and many other researchers. It has led to a beautiful and fundamental theory that is so important that it is covered by all standard textbooks in combinatorial optimization nowadays. For instance, see [1] for a thorough treatment of the subject.

When $\delta_{\max }(A)>1$, then surprisingly little is known.

Bonifas et al. showed in [2] that for a bounded polyhedron $P=\{x \in$ $\left.{ }^{n}: A x \leqslant b\right\}$ its (combinatorial) diameter is bounded in the order of $O\left(\delta_{\max }(A)^{2}\right.$. $\left.n^{3.5} \cdot \log \left(n \cdot \delta_{\max }(A)\right)\right)$. This improves an important result of Dyer and Frieze 3] that applies to TU-matrices.

Veselov and Chirkov (2009) showed in [4, how (2) can be solved polynomially in $m$ and $n$ and the encoding size of the data when $\delta_{\max }(A) \leq 2$ and no $(n \times n)$ submatrices are singular.

There exists a dynamic programming approach to solve (10) by Papadimitriou [5], see also [1], Part IV, Section 18.6: Let $\Delta(A, b)$ be an upper bound on the absolute values of $A$ and $b$. Then, if (11) is feasible and bounded, it has an optimal solution with components bounded by $U:=(n+1)(m \cdot \Delta(A, b))^{m}$.

The dynamic program is a maximum weight path problem on a properly defined (acyclic) graph. The optimum solution can be found in time

$$
\mathcal{O}(|V|)=\mathcal{O}\left(2^{m} \cdot n^{m+1} \cdot(m \cdot \Delta(A, b))^{m^{2}} \cdot \Delta(A, b)^{m}\right) .
$$

We show how to avoid a dependence of the running time on the largest absolute value of an entry in $b$ : For fixed $m$, an integer program can be solved in time polynomially bounded by $n$ and the largest absolute value $\Delta$ of an entry in $A$. This result is one important ingredient to solve the optimization problem (2) in polynomial-time for any constant values of $\delta_{\max }(A)$, provided that $A$ has no singular $(n \times n)$-submatrices and $\operatorname{rank}(A)=n$. It turns out that the condition that all $(n \times n)$-sub-determinants shall be non-zero imposes very harsh restrictions on $A$. In particular, $A$ can have at most $n+1$ rows provided that $n$ exceeds a certain constant.

\section{Dynamic Programming Revisited}

\subsection{The Pure Integer Case}

We show that one can solve problem (1) in time polynomial in $n, \Delta$ and $\log \left(\max _{i}\left\{\left|b_{i}\right|\right\}\right)$ where $\Delta=\max _{i, j}\left\{\left|A_{i, j}\right|\right\}$. This is an improvement over Papadimitriou's approach [5], as we eliminate the unary dependency on $b$. For $S \subseteq\{1, \ldots, n\}$, let $A_{S}$ denote the matrix stemming from $A$ by the columns indexed by $S$.

Lemma 1. If the integer program (1) is feasible and bounded, there exists an optimal solution $x^{*} \in \mathbb{Z}^{n}$ where at least $n-m$ components of $x^{*}$ are bounded by 
$(m+2) \cdot(m \cdot \Delta)^{m}$. Furthermore, the columns of A corresponding to components of $x^{*}$ that are larger than $(m+2) \cdot(m \cdot \Delta)^{m}$ are linearly independent.

The proof of this Lemma is in Appendix A Once this Lemma is shown, we have the following result.

Theorem 2. There exists an algorithm that solves the integer programming problem (11) in time bounded by

$$
\mathcal{O}\left(2^{2 m} \cdot \Delta^{m^{3}+3 m^{2}+2 m} \cdot n^{m^{3}+5 m^{2}+6 m+1}\right) .
$$

Proof. We assume that problem is feasible and bounded. Let $x^{\star}$ be the optimal solution as defined in Lemma 1 and let $S \subseteq\{1, \ldots, n\}$ be the set of indices of the components of $x^{*}$ that are bounded by $(m+2) \cdot(m \cdot \Delta)^{m}$. By $\bar{S}$, we denote the complement of $S$. Now, let

$$
b^{\prime \prime}:=\sum_{j \in S} x_{j}^{*} A \cdot, j \text { and } b^{\prime}:=b-b^{\prime \prime} .
$$

It follows that $x_{S}^{*}$ is an optimal solution of the integer program

$$
\max \left\{\sum_{j \in S} c_{j} x_{j}: \sum_{j \in S} x_{j} A \cdot, j=b^{\prime \prime}, x \in \mathbb{Z}_{\geqslant 0}^{S}\right\},
$$

and $x_{\frac{*}{S}}$ is the optimal solution of the integer program

$$
\max \left\{\sum_{j \in \bar{S}} c_{j} x_{j}: \sum_{j \in \bar{S}} x_{j} A_{\cdot, j}=b^{\prime}, x \in \mathbb{Z}_{\geqslant 0}^{\bar{S}}\right\} .
$$

Since $\left\|b^{\prime \prime}\right\|_{\infty} \leqslant \Delta \cdot n \cdot(m+2) \cdot(m \cdot \Delta)^{m}$, the integer programming problem (3) can be solved with Papadimitriou's algorithm [5] in time $\mathcal{O}\left(2^{m} \cdot \Delta^{m^{2}+m}\right.$. $\left.n^{m^{2}+2 m+1} \cdot m^{2 m^{2}+m} \cdot(m \cdot \Delta)^{m^{3}+m^{2}}\right)$.

Since the columns of $A_{\bar{S}}$ are linearly independent, $x_{\bar{S}}^{*}$ is the unique solution of the system of equations

$$
\sum_{j \in \bar{S}} x_{j} A_{\cdot, j}=b^{\prime},
$$

which can be found by using Gaussian elimination.

The algorithm starts by enumerating all possible

$$
\mathcal{O}\left(2^{m} \cdot \Delta^{m} \cdot n^{m} \cdot(m+2)^{m} \cdot(m \cdot \Delta)^{m^{2}}\right)
$$

vectors $b^{\prime \prime}$ and then proceeds by enumerating all $\left(\begin{array}{c}n \\ m\end{array}\right)=O\left(n^{m}\right)$ components of $x^{*}$ whose absolute value might be larger than $(m+2) \cdot(m \cdot \Delta)^{m}$ in the optimal solution $x^{*}$. Then, one solves the integer program (3) with Papadimitriou's algorithm and the integer program (4) using Gaussian elimination. 
Altogether this yields a running time of

$$
\begin{gathered}
\mathcal{O}\left(2^{m} \cdot \Delta^{m} \cdot n^{m} \cdot(m+2)^{m} \cdot(m \cdot \Delta)^{m^{2}} \cdot n^{m} \cdot 2^{m}\right. \\
\left.\Delta^{m^{2}+m} \cdot n^{m^{2}+2 m+1} \cdot m^{2 m^{2}+m} \cdot(m \cdot \Delta)^{m^{3}+m^{2}}\right) .
\end{gathered}
$$

We can assume that $m \leqslant n$ and obtain the running time

$$
\mathcal{O}\left(2^{2 m} \cdot \Delta^{m^{3}+3 m^{2}+2 m} \cdot n^{m^{3}+5 m^{2}+6 m+1}\right) .
$$

Proof of Lemma 1. We assume that the objective function vector $c$ is nondegenerate in the following sense: We suppose that $c^{T} y \neq 0$ for each integral vector $y \neq 0$ with $\|y\|_{\infty} \leqslant(m+2) \cdot(m \cdot \Delta)^{m}$. This can be achieved without loss of generality with standard perturbation, i.e., $c:=c+\left(\varepsilon, \varepsilon^{2}, \ldots, \varepsilon^{n}\right)$ for $\varepsilon>0$ small.

Let $x^{*}$ be an optimal solution and let $S \subseteq\{1, \ldots, n\}$ be a subset of indices for which $x_{s}^{*} \geqslant(m+2) \cdot(m \cdot \Delta)^{m}$ for each $s \in S$. If the columns of $A \cdot, S$ are not linearly independent, then there exists a non-zero integral $d \in \mathbb{Z}^{|S|}, d \neq 0$, with $A \cdot, S \cdot d=0$. We can assume that the support of $d$ fulfills $|\operatorname{supp}(d)| \leq m+1$. Then, as noted in the introduction, there exists a feasible solution to $A \cdot, \cdot x=0$, $x \in \mathbb{Z}^{|S|}$, with $\|d\|_{\infty} \leqslant(m+2)(m \cdot \Delta)^{m}$. Without loss of generality, $c^{T} d>0$, using our modified objective function. But then, $\left(x_{S}^{*}+d, x_{\bar{S}}^{*}\right)$ is a feasible solution with better objective function, which is a contradiction. Consequently, the number of components of $x^{*}$ exceeding $(m+2)(m \cdot \Delta)^{m}$ is bounded by $m$ and the corresponding columns of $A$ are linearly independent.

\subsection{Extensions to the Mixed Integer Setting}

This section is devoted to generalizations of Lemma 1 and Theorem 2 in order to apply the idea from the previous section to mixed-integer optimization problems of the form

$$
\max \left\{c^{T} x+d^{T} y: A x+B y=b, x, y \geqslant 0, x \in \mathbb{Z}^{n}, y \in{ }^{l}\right\},
$$

where, as before, $A \in \mathbb{Z}^{m n}$ with upper bound $\Delta$ on the absolute values of $A$, $b \in \mathbb{Z}^{m}, c \in \mathbb{Z}^{n}$ and $d \in \quad$.

If we view problem (5) as a parametric integer problem in variables $x$ only, then Lemma 1 is applicable. This observation directly leads us to a mixedinteger version of Lemma 1 .

Lemma 3. If the mixed-integer program (5) has an optimal solution, then it has an optimal solution $\left(x^{*}, y^{*}\right)$ such that $x^{*} \in \mathbb{Z}^{n}$, where at least $n-m$ components of $x^{*}$ are bounded by $(m+2) \cdot(m \cdot \Delta)^{m}$. Furthermore, the columns of $A$ corresponding to components of $x^{*}$ that are larger than $(m+2) \cdot(m \cdot \Delta)^{m}$ are linearly independent. 
With this Lemma, we are prepared to prove a mixed-integer version of Theorem 2 In the special case when $m$ is a constant, this result gives rise to a polynomial-time algorithm for solving the mixed-integer optimization problem (5).

Theorem 4. There exists an algorithm that solves the mixed-integer programming problem (5) in time bounded by

$$
\mathcal{O}\left(2^{2 m} \cdot \Delta^{m^{3}+3 m^{2}+2 m} \cdot n^{m^{3}+5 m^{2}+6 m+1}\right) \cdot \kappa(m, l, \Delta),
$$

where $\kappa(m, l, \Delta)$ is the worst case running time for solving a mixed-integer optimization problem of the type (5) with $m$ integer variables and $l$ continuous variables.

Proof. Let $\left(x^{*}, y^{*}\right)$ be an optimal solution of problem (5) satisfying Lemma 3 . By $S \subseteq\{1, \ldots, n\}$ we denote the indices of the components of $x^{*}$ that are bounded by $(m+2) \cdot(m \cdot \Delta)^{m}$. Furthermore let

$$
b^{\prime \prime}:=\sum_{j \in S} x_{j}^{*} A \cdot, j \text { and } b^{\prime}:=b-b^{\prime \prime} .
$$

Then, $x_{S}^{*}$ is an optimal solution of the pure integer program

$$
\max \left\{\sum_{j \in S} c_{j} x_{j}: \sum_{j \in S} x_{j} A_{\cdot, j}=b^{\prime \prime}, x \in \mathbb{Z}_{\geqslant 0}^{|S|}\right\},
$$

and $\left(x_{S}^{*}, y^{*}\right)$ is the optimal solution of the mixed-integer program

$$
\begin{aligned}
\max & \left\{\sum_{j \in \bar{S}} c_{j} x_{j}+\sum_{j=1}^{l} d_{j} y_{j}:\right. \\
& \left.\sum_{j \in \bar{S}} x_{j} A_{\cdot, j}+\sum_{j=1}^{l} y_{j} B \cdot, j=b^{\prime}, x \in \mathbb{Z}_{\geqslant 0}^{|\bar{S}|}, y \geqslant 0\right\} .
\end{aligned}
$$

As in the previous section, the algorithm first enumerates all possible

$$
\mathcal{O}\left(2^{m} \cdot \Delta^{m} \cdot n^{m} \cdot(m+2)^{m} \cdot(m \cdot \Delta)^{m^{2}}\right)
$$

vectors $b^{\prime \prime}$, which satisfy

$$
\left\|b^{\prime \prime}\right\|_{\infty} \leqslant \Delta \cdot n \cdot(m+2) \cdot(m \cdot \Delta)^{m} .
$$

Next, one proceeds by enumerating all $\left(\begin{array}{c}n \\ m\end{array}\right)=O\left(n^{m}\right)$ components of $x^{*}$ whose absolute value might be larger than $(m+2) \cdot(m \cdot \Delta)^{m}$ in the optimal solution $x^{*}$. The corresponding integer programming problem (6) can be solved with Papadimitriou's algorithm in time $\mathcal{O}\left(2^{m} \cdot \Delta^{m^{2}+m} \cdot n^{m^{2}+2 m+1} \cdot m^{2 m^{2}+m}\right.$. $\left.(m \cdot \Delta)^{m^{3}+m^{2}}\right)$. Since the columns of $A_{\bar{S}}$ are linearly independent, $|\bar{S}| \leqslant m$. Therefore, the mixed-integer program (17) can be solved in time $\kappa(m, l, \Delta)$.

Altogether, this yields the proposed running time. 


\section{Application to integer programs with non-zero and bounded sub-determinants}

Let us now use Theorem 2 to prove that (2) can be solved in polynomial-time under the assumption that $A$ has rank $n, A$ has no singular $(n \times n)$-submatrices and $\delta_{\max }(A) \leq \delta$ for a constant $\delta<\infty$. Our plan is as follows:

First, we permute the rows of $A$ and transform the permuted matrix into Hermite Normal Form to obtain the representation illustrated in (8). This can be accomplished in polynomial-time (cf. [6]). Then, we show that the entries of $A$ are bounded by a constant $C(\delta)$ depending solely on $\delta$ and that for $n$ large enough, $A$ can have at most $n+1$ rows. Using these results, we find an efficient algorithm for (2): We reformulate (2) as a program of the form given in (1) with a matrix whose number of rows is bounded by a constant which only depends on $\delta$. We then apply Theorem 2 to get a polynomial running-time algorithm.

By permuting the rows of $A$, we may assume without loss of generality that $\operatorname{det}\left(A_{1: n,}\right) \neq 0$, where $A_{1: n,}$, is the uppermost $(n \times n)$-submatrix of $A$. Let $U$ be the unimodular matrix such that $A_{1: n}, U$ is in Hermite Normal Form (cf. 11, Part II, Chapters 4 and 5). Note that as $U^{-1} \in \mathbb{Z}^{n \times n}, A U$ has the same $(n \times n)$-sub-determinants as $A$ and that $U$ can be calculated in polynomial-time (in $m, n$ and the encoding size of $A$ ) and is polynomially bounded in the size of $A$ (cf. [6] or [1, Part II, Chapter 5.2). After a change of variables from $x$ to $U^{-1} x$, we can assume from now on that $A$ is a lower triangular matrix with diagonal entries

$$
\left(1, \ldots, 1, \delta_{1}, 1, \ldots, 1, \delta_{2}, 1, \ldots, 1, \delta_{k}, 1, \ldots, 1, A_{n, n}\right) .
$$

Moreover, we have that $\delta_{i} \leq \delta$ for all $i \in\{1, \ldots, k\}$ and for all $1 \leq j<i \leq n$ we have $A_{i, j}<A_{i, i}$.

Our next step is to simplify $A$ further. To this end, we apply row permutations and column permutations iteratively: For $i \in\{1, \ldots, k\}$, assume that after row and column permutations, the rows corresponding to the diagonal entries $\delta_{i+1}, \ldots, \delta_{k}$ are at positions $n-i-1, \ldots, n$. Let $A^{\delta_{i}}$ be the row corresponding to $\delta_{i}$. Then, exchange rows $A^{\delta_{i}}$ and $A_{n-(i+1)}$, as well as the column containing $\delta_{i}$ with column $n-(i+1)$. This leads to a representation of the matrix $A$ as follows: 


$$
A=\left[\begin{array}{ccccccc}
1 & & & & & & \\
& \ddots & & & & & \\
* & \cdots & * & \delta_{1} & & & \\
\vdots & & & \ddots & \ddots & & \\
* & \cdots & \cdots & \cdots & * & \delta_{k} & \\
A_{n, 1} & \cdots & \cdots & \cdots & \cdots & A_{n, n-1} & A_{n, n} \\
A_{n+1,1} & \cdots & \cdots & \cdots & \cdots & A_{n+1, n-1} & A_{n+1, n} \\
\vdots & \vdots & \vdots & \vdots & \vdots & \vdots & \vdots \\
A_{m, 1} & \cdots & \cdots & \cdots & \cdots & A_{m, n-1} & A_{m, n}
\end{array}\right] .
$$

All entries denoted by $*$ are numbers between 0 and the diagonal entry of the same row.

The submatrix consisting of the first $n$ rows has a determinant that is bounded by $\delta$. This allows us to conclude that $\left|\delta_{1}\right| \cdots\left|\delta_{k}\right| \cdot\left|A_{n, n}\right| \leq \delta$ and thus $k \leq \log _{2}(\delta)$.

We will now make use of two Lemmas. The corresponding proofs are postponed to Appendix $\mathrm{A}$.

Lemma 5. The entries in $A$ are bounded by a constant $C(\delta)$ which only depends on $\delta$.

Remark 6. Lemma 5 also holds in the case where $A$ has singular $(n \times n)$ submatrices.

Lemma 7. Let $n>(2 C(\delta)+1)^{\log _{2} \delta+3}+\log _{2} \delta$. Then, $A$ has at most $n+1$ rows.

We are now prepared to prove the main result of this section.

Theorem 8. There exists an algorithm that solves problem (2) in time polynomially bounded by $m, n, \delta$ and the encoding size of the input data.

Proof of Theorem 8 . If $n \leqslant(2 C(\delta)+1)^{\log _{2} \delta+3}+\log _{2} \delta$, then by using Lenstra's algorithm (cf. [7]), the corresponding integer program can be solved in polynomialtime.

Otherwise, $n>(2 C(\delta)+1)^{\log _{2} \delta+3}+\log _{2} \delta$. By Lemma 7, $A$ has at most $n+1$ rows. Furthermore, in view of (8), we may assume that $A$ is of the form

$$
A=\left[\begin{array}{cc}
-\mathcal{I} & 0 \\
\widetilde{A} & \widehat{A}
\end{array}\right]
$$


where $\mathcal{I}$ denotes the $(n-k-1)$-dimensional identity matrix, $\mathbf{0}=\{0\}^{(n-k-1) \times(k+1)}$,

$$
\widetilde{A}:=\left[\begin{array}{ccc}
* & \cdots & * \\
\vdots & & \vdots \\
* & \cdots & * \\
\alpha_{1} & \cdots & \alpha_{n-k-1} \\
\beta_{1} & \cdots & \beta_{n-k-1}
\end{array}\right] \in \mathbb{Z}^{(k+2) \times(n-k-1)}
$$

and

$$
\widehat{A}:=\left[\begin{array}{cccc}
\delta_{1} & & & \\
\ddots & \ddots & & \\
\cdots & * & \delta_{k} & \\
\alpha_{n-k} & \ldots & \alpha_{n-1} & \alpha_{n} \\
\beta_{n-k} & \ldots & \beta_{n-1} & \beta_{n}
\end{array}\right] \in \mathbb{Z}^{(k+2) \times(k+1)} .
$$

It holds that $\delta \geq \delta_{i}>0$ and by Lemma 5 , $\left|A_{i j}\right| \leqslant C(\delta)$ for all $1 \leq i \leq n+1$, $1 \leq j \leq n$.

We denote $\bar{A}:=\left[\begin{array}{ll}\widetilde{A} & \widehat{A}\end{array}\right]$. For a vector $x \in{ }^{n}$, let $\widetilde{x} \in \mathbb{Z}^{n-k-1}$ be the first $n-k-1, \widehat{x} \in \mathbb{Z}^{k+1}$ be the last $k+1$ components of $x$. Thus, $\bar{A} x=\widetilde{A} \widetilde{x}+\widehat{A} \widehat{x}$. We write $x=\left(\begin{array}{l}\widetilde{x} \\ \widehat{x}\end{array}\right)$. Similarly, we write $b=\left(\begin{array}{l}\tilde{b} \\ \bar{b}\end{array}\right)$, where $\widetilde{b} \in \mathbb{Z}^{n-k-1}$ and $\bar{b} \in \mathbb{Z}^{k+2}$, such that $A x \leq b \Leftrightarrow \widetilde{x} \geq \widetilde{b}, \bar{A} x \leq \bar{b}$.

$A x \leq b, x \in \mathbb{Z}^{n}$ can then be reformulated as $\bar{A} x \leq \bar{b}, \tilde{x} \geq \widetilde{b}, x \in \mathbb{Z}^{n}$, which in turn can be restated as

$$
\begin{aligned}
\bar{A} y & \leq \bar{b}-\bar{A}\left(\begin{array}{c}
\widetilde{b} \\
0
\end{array}\right), \\
\widetilde{y} & \geq 0,
\end{aligned}
$$

where $y:=x-\left(\begin{array}{c}\widetilde{b} \\ 0\end{array}\right)$.

This reformulation leads to the following maximization problem:

$$
\max \left\{c^{T} x: \bar{A} x \leq \bar{b}, x=\left(\begin{array}{c}
\widetilde{x} \\
\widehat{x}
\end{array}\right), \widetilde{x} \geq 0, x \in \mathbb{Z}^{n}\right\} .
$$

To arrive at a problem of the form (11), we apply a standard technique: We introduce new variables $\widehat{x}_{i}^{+}:=\max \left\{\widehat{x}_{i}, 0\right\}$ and $\widehat{x}_{i}^{-}:=\min \left\{\widehat{x}_{i}, 0\right\}$ as well as slack variables $z \geq 0$ and reformulate (9) as

$$
\begin{aligned}
& \max \left[\begin{array}{llll}
\widetilde{c}^{T} & \widehat{c}^{T} & -\widehat{c}^{T} & \mathbf{0}^{T}
\end{array}\right]\left[\begin{array}{llll}
\widetilde{x} & \widehat{x}^{+} & \widehat{x}^{-} & z
\end{array}\right]^{T} \\
& \text { s.t. }\left[\begin{array}{llll}
\widetilde{A} & \widehat{A} & -\widehat{A} & \mathcal{I}
\end{array}\right]\left[\begin{array}{llll}
\widetilde{x} & \widehat{x}^{+} & \widehat{x}^{-} & z
\end{array}\right]^{T}=\bar{b} \text {, } \\
& \widetilde{x}, \widehat{x}^{+}, \widehat{x}^{-}, z \geq 0,
\end{aligned}
$$


where $c=\left(\begin{array}{l}\widetilde{c} \\ \widehat{c}\end{array}\right)$. Here, $\mathbf{0} \in \mathbb{Z}^{k+2}$ denotes the $(k+2)$-dimensional zero vector and $\mathcal{I}$ is the $(k+2) \times(k+2)$-identity matrix.

Recall that $k \leq \log _{2}(\delta)$. Hence, the matrix $\left[\begin{array}{llll}\widetilde{A} & \widehat{A} & -\widehat{A} & \mathcal{I}\end{array}\right] \in \mathbb{Z}^{(k+2) \times(n+2 k+3)}$ in (10) has at most $\log _{2} \delta+2$ rows and at most $n+2 \log _{2} \delta+3$ columns. Furthermore, in view of Lemma 5 , each entry is bounded by $C(\delta)$. We can therefore apply Theorem 2 This gives the desired result, where the overall running time is bounded by

$$
\mathcal{O}\left(n^{\left(\log _{2} \delta+2\right)^{3}+5\left(\log _{2} \delta+2\right)^{2}+6\left(\log _{2} \delta+2\right)+1}\right) .
$$

\section{A Technical Proofs}

Proof of Lemma [5. We assume that $A$ is in the form (8). The first $n$ rows are part of the Hermite Normal Form. Hence, by definition, they fulfill the statement. Let $\alpha=\left[\alpha_{1}, \ldots, \alpha_{n}\right]$ be any other row of $A$.

Let $B_{0}:=\delta, q:=\left\lceil\log _{2} \delta\right\rceil$ and for $i \in\{1, \ldots, q\}$, consider the increasing sequence $B_{i}:=\delta+\sum_{l=0}^{i-1} B_{l} \delta^{\log _{2} \delta}\left(\log _{2} \delta\right)^{\left(\log _{2} \delta\right) / 2}$. We show that all entries of $\alpha$ are bounded by the constant $B_{q}$ as follows.

Let $A_{i}$ denote the square submatrix of $A$ that consists of the first $n$ rows, except for the $i$-th row, which is replaced by $\alpha$. Then, since $\left|\operatorname{det}\left(A_{n}\right)\right|=$ $\delta_{1} \ldots \delta_{k} \cdot \alpha_{n}$, it follows that $\left|\alpha_{n}\right| \leq \delta=B_{0}$.

Case i) $i \geq n-k$ :

Consider $\alpha_{i}$ and assume that it holds that $B_{n-j} \geq\left|\alpha_{j}\right|$ for all $n \geq j>i$. We can express $\left|\operatorname{det}\left(A_{i}\right)\right|$ as follows.

$$
\left|\delta_{1}\right| \cdots\left|\delta_{r-1}\right||\operatorname{det} \underbrace{\left[\begin{array}{ccccc}
\alpha_{i} & & \cdots & & \alpha_{n} \\
* & \delta_{r+1} & & & \\
* & * & \ddots & & \\
\vdots & \vdots & * & \delta_{k} & \\
A_{n, i} & & \ldots & & A_{n, n}
\end{array}\right]}_{=: \bar{A}}| .
$$

Let $\bar{A}^{j}$ be the matrix $\bar{A}$ without the first row and without column $j$. Then,

$$
\begin{aligned}
\delta & \geq|\operatorname{det} \bar{A}|=\left|\alpha_{i} \operatorname{det} \bar{A}^{1}+\sum_{l=2}^{n-i+1}(-1)^{l+1} \alpha_{l+i-1} \operatorname{det} \bar{A}^{l}\right| \\
& \geq\left|\alpha_{i} \operatorname{det} \bar{A}^{1}\right|-\sum_{l=2}^{n-i+1}\left|\alpha_{l+i-1} \operatorname{det} \bar{A}^{l}\right|,
\end{aligned}
$$


and thus

$$
\left|\alpha_{i}\right| \leq \frac{1}{\left|\operatorname{det} \bar{A}^{1}\right|}\left(\delta+\sum_{l=2}^{n-i+1}\left|\alpha_{l+i-1} \operatorname{det} \bar{A}^{l}\right|\right) .
$$

Furthermore, $1 \leq\left|\operatorname{det} \bar{A}^{1}\right| \leq \delta$.

Since the absolute value of each entry in $\bar{A}^{j}$ is bounded by $\delta$, we can apply the Hadamard inequality [8] to obtain $\left|\operatorname{det} \bar{A}^{j}\right| \leq \delta^{n-i}(n-i)^{(n-i) / 2} \leq$ $\delta^{\log _{2} \delta}\left(\log _{2} \delta\right)^{\left(\log _{2} \delta\right) / 2}$. This provides us with the bound

$$
\left|\alpha_{i}\right| \leq \delta+\sum_{l=2}^{n-i+1} B_{n-l-i+1} \delta^{\log _{2} \delta}\left(\log _{2} \delta\right)^{\left(\log _{2} \delta\right) / 2}=B_{n-i} \leq B_{k} .
$$

Thus $\left|\alpha_{i}\right| \leq B_{k} \leq B_{q}$.

Case ii) $i<n-k$ :

Similar to the previous case, we can express $\left|\operatorname{det}\left(A_{i}\right)\right|$ as

$$
|\operatorname{det} \underbrace{\left[\begin{array}{ccccc}
\alpha_{i} & \alpha_{n-k} & \cdots & \cdots & \alpha_{n} \\
* & \delta_{1} & & & \\
* & * & \ddots & & \\
\vdots & \vdots & & \delta_{k} & \\
A_{n, i} & A_{n, n-k} & \cdots & \cdots & A_{n, n}
\end{array}\right]}_{=: \bar{A}}| .
$$

Let $\bar{A}^{j}$ be $\bar{A}$ without the first row and column $j$, so that

$$
\begin{aligned}
\delta & \geq|\operatorname{det} \bar{A}|=\left|\alpha_{i} \operatorname{det} \bar{A}^{1}+\sum_{l=2}^{k+2}(-1)^{l+1} \alpha_{l+n-k-2} \operatorname{det} \bar{A}^{l}\right| \\
& \geq\left|\alpha_{i} \operatorname{det} \bar{A}^{1}\right|-\sum_{l=2}^{k+2}\left|\alpha_{l+n-k-2} \operatorname{det} \bar{A}^{l}\right|,
\end{aligned}
$$

and

$$
\left|\alpha_{i}\right| \leq \frac{1}{\left|\operatorname{det} \bar{A}^{1}\right|}\left(\delta+\sum_{l=2}^{k+2}\left|\alpha_{l+n-k-2} \operatorname{det} \bar{A}^{l}\right|\right) .
$$

We arrive at the bound

$$
\left|\alpha_{i}\right| \leq \delta+\sum_{l=2}^{k+2} B_{k+2-l} \delta^{\log _{2} \delta}\left(\log _{2} \delta\right)^{\left(\log _{2} \delta\right) / 2} \leq B_{q}
$$

This completes the proof by letting $C(\delta):=B_{q}$. 
Proof of Lemma 7. Let $A$ be defined as illustrated in (8). Recall that $A$ has no singular $(n \times n)$-submatrices. For the purpose of deriving a contradiction, assume that $n>(2 C(\delta)+1)^{\log _{2} \delta+3}+\log _{2} \delta$ and that $A$ has precisely $n+2$ rows. Let $\tilde{A}$ be the matrix $A$ without rows $i$ and $j$, where $i, j<n-k, i \neq j$. Observe that

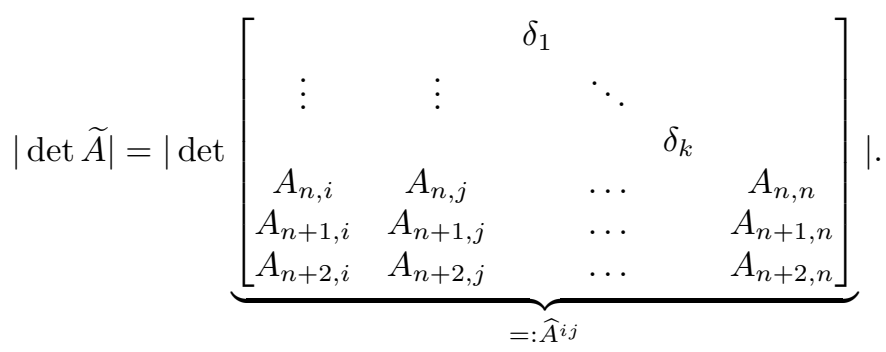

$\widehat{A}^{i j}$ is a $(k+3) \times(k+3)$-matrix. Its determinant cannot be zero. This implies that the first two columns of $\widehat{A}^{i j}$ must be different for each choice of $i$ and $j$.

From Lemma 5. it follows that the absolute value of any entry of $\widehat{A}^{i j}$ is bounded by $C(\delta)$. Therefore, the first two columns are in $\{-C(\delta), \ldots, C(\delta)\}^{k+3}$. Since $k \leq \log _{2} \delta$, there exist at most $(2 C(\delta)+1)^{\log _{2} \delta+3}$ such vectors. Consequently, as $n>(2 C(\delta)+1)^{\log _{2} \delta+3}+\log _{2} \delta$, there must exist two indices $i \neq j \in\{1, \ldots, n-k-1\}$ such that $\operatorname{det}\left(\widehat{A}^{i j}\right)=0$. This contradicts that there are no singular $(n \times n)$-submatrices within $A$. The statement follows.

\section{References}

[1] A. Schrijver, Theory of Linear and Integer Programming, John Wiley and Sons, NY, 1986.

[2] N. Bonifas, M. Di Summa, F. Eisenbrand, N. Hähnle, M. Niemeier, On sub-determinants and the diameter of polyhedra, Discrete \& Computational Geometry 52 (1) (2014) 102-115. doi:10.1007/s00454-014-9601-x.

[3] M. Dyer, A. Frieze, Random walks, totally unimodular matrices, and a randomised dual simplex algorithm, Mathematical Programming 64 (1-3) (1994) 1-16. doi:10.1007/BF01582563.

[4] S. I. Veselov, A. J. Chirkov, Integer program with bimodular matrix, Discrete Optimization 6 (2) (2009) 220-222. doi:10.1016/j.disopt.2008.12.002

[5] C. H. Papadimitriou, On the complexity of integer programming., J. ACM 28 (4) (1981) 765-768. doi:10.1145/322276.322287.

[6] M. A. Frumkin, An algorithm for the reduction of a matrix of integers to triangular form with power complexity of the computations (in Russian), Ekonomika i Matematicheskie Metody 12 (1976) 173-178. 
[7] H. W. Lenstra, Integer programming with a fixed number of variables, Mathematics of operations research 8 (4) (1983) 538-548. doi:10.1287/moor.8.4.538.

[8] J. Hadamard, Rèsolution d'une question relative aux dèterminants, Bulletin des Sciences Mathématiques 2 (17) (1893) 240-246. 